\title{
Semilinear Elliptic Systems With Exponential Nonlinearities in Two Dimensions*
}

\author{
Djairo G. de Figueiredo
}

IMECC - Departamento de Matemática, Universidade Estadual de Campinas, 13081 Campinas, S.P., Brazil e-mail: djairo@ime.unicamp.br

\section{João Marcos do Ó}

CCEN - Departamento de Matemática, Universidade Federal da Paraíba, 58059.900 João Pessoa, Pb, Brazil e-mail: jmbo@mat.ufpb.br

\section{Bernhard Ruf}

Dipartimento di Matematica, Università degli Studi, Via Saldini 50, 20133 Milano, Italy e-mail: ruf@mat.unimi.it

Received 17 March 2006

Communicated by Shair Ahmad

Abstract
We establish a priori bounds for positive solutions of semilinea
\[ \left\{\begin{array}{r}-\Delta u=g(x, v), \text { in } \Omega \\ -\Delta v=f(x, u), \text { in } \Omega \\ u>0, v>0 \text { in } \Omega \\ u=v=0 \text { on } \partial \Omega\end{array}\right. \]

where $\Omega$ is a bounded and smooth domain in $\mathbb{R}^{2}$. We obtain results concerning such bounds when $f$ and $g$ depend exponentially with respect to $u$ and $v$.

*Work partially supported by PADCT/CT-INFRA/CNPq/MCT, Grant \# 620120/2004-5 and Millennium Institute for the Global Advancement of Brazilian Mathematics-IM-AGIMB. 
1991 Mathematics Subject Classification. 35J60, 35B45.

Key words. Elliptic systems, A priori estimates.

\section{Introduction}

In this paper we establish the existence of a priori bounds for positive solutions of semilinear elliptic systems of the form

$$
\left\{\begin{array}{c}
-\Delta u=g(x, v), \text { in } \Omega \\
-\Delta v=f(x, u), \text { in } \Omega \\
u>0, v>0 \text { in } \Omega \\
u=v=0 \text { on } \partial \Omega
\end{array}\right.
$$

where $\Omega$ is a bounded domain in $\mathbb{R}^{2}$ with smooth boundary $\partial \Omega$, and $\Delta$ is the Laplace operator. Let $\lambda_{1}$ denote the first eigenvalue of $\left(-\Delta, H_{0}^{1}(\Omega)\right)$. Throughout the paper the nonlinearities satisfy the following conditions:

(i) $\quad f, g: \Omega \times \mathbb{R} \rightarrow \mathbb{R}^{+}$are continuous.

(ii) $\liminf _{t \rightarrow \infty} \frac{f(x, t)}{t} \geq a_{1}>0$ and $\liminf _{t \rightarrow \infty} \frac{g(x, t)}{t} \geq a_{2}>0$, with $a_{1} a_{2}>\lambda_{1}^{2}$.

(iii) $\frac{\partial}{\partial t} f(x, t) \geq 0$ and $\frac{\partial}{\partial t} g(x, t) \geq 0$ in $\Omega_{r} \times \mathbb{R}$, where, for some $r>0$,

$$
\Omega_{r}:=\{x \in \bar{\Omega}: \operatorname{dist}(x, \partial \bar{\Omega}) \leq r\} .
$$

We note that condition (iii) allows the use of the Maximum Principle for cooperative systems; this is a basic tool to apply the Moving Planes technique (cf. [6], [12]).

To start with, we consider here solutions in the sense of distributions, more precisely, we assume that $u, v, f(x, v), g(x, u) \in L^{1}(\Omega)$ and

$$
-\int_{\Omega} u \Delta \varphi d x=\int_{\Omega} g(x, v) \varphi d x \text { and }-\int_{\Omega} v \Delta \varphi d x=\int_{\Omega} f(x, u) \varphi d x, \varphi \in C_{0}^{2}(\bar{\Omega}),
$$

where $C_{0}^{2}(\bar{\Omega})$ is the class of $C^{2}$ functions in $\bar{\Omega}$ which vanish on the boundary $\partial \Omega$.

Before stating our main results on a priori bounds, we state a result on the regularity of the distribution solutions of (1.1). For this, a growth assumption on only one of the nonlinearities suffices. Making the following hypotheses

$$
\begin{aligned}
& \left(H_{1}\right) \quad f(x, t) \leq c e^{p t}, \text { for some constants } c>0 \text { and some } p>0 \\
& \left(H_{1}^{\prime}\right) \quad g(x, t) \leq c e^{q t}, \text { for some constants } c>0 \text { and some } q>0
\end{aligned}
$$

we have 
Theorem 1.1 (Regularity of distribution solutions) Assume $\left(H_{1}\right)$ (or $\left(H_{1}^{\prime}\right)$ ). Then the distribution solutions of system (1.1) are in fact in $L^{\infty}(\Omega)$.

It follows from Theorem 1.1 that, for any solution $(u, v)$ of system (1.1), $\int_{\Omega} g(x, u) d x<\infty, \int_{\Omega} f(x, u) d x<\infty$. Our next result states that there is a uniform bound for those integrals. For that matter, due to the fact that we are considering nonautonomous problems, we need in the theorems below geometric assumptions concerning the behavior of $f$ and $g$ near the boundary. So,

$\left(H_{2}\right)$ (For the case of a convex domain) There exist $r, \delta>0$ such that $g(\cdot, t), f(\cdot, t) \in C^{1}\left(\Omega_{r}\right)$ for all $t \geq 0$, and

$$
\nabla_{x} g(x, t) \cdot \theta \leq 0 \text { and } \nabla_{x} f(x, t) \cdot \theta \leq 0
$$

for all $x \in \Omega_{r}, t \geq 0$, and unit vectors $\theta$ such that $|\theta-\nu(x)|<\delta ; \nu(x)$ denotes the unit external normal to $\partial \Omega$ in the point $x$.

With assumption $\left(\mathrm{H}_{2}\right)$ one can use the Moving Planes technique to get bounds for the functions $u$ and $v$ near the boundary. On the other hand, if $\Omega$ is not convex we use the Kelvin transform as in [7] and [5] to reduce the problem to a situation as in the convex case. So we follow [5] and assume

$\left(H_{3}\right)$ (For the case of a general domain) There exists $r, C>0$ such that $g(\cdot, t), f(\cdot, t) \in C^{1}\left(\Omega_{r}\right)$ for all $t \geq 0$ and

$$
\left|\nabla_{x} g(x, t)\right| \leq C g(x, t) \text { and }\left|\nabla_{x} f(x, t)\right| \leq C f(x, t), \text { for all } x \in \Omega_{r}, t \geq 0 .
$$

Theorem 1.2 (Uniform Estimates) Assume $\left(H_{1}\right)$ (or $\left(H_{1}^{\prime}\right)$ ), and $\left(H_{2}\right)$ or $\left(H_{3}\right)$ Then there exists a positive constant $C$, depending only on $f, g$ and $\Omega$, such that

$$
\int_{\Omega} g(x, v) d x \leq C, \int_{\Omega} f(x, u) d x \leq C
$$

for all $(u, v)$ solution of (1.1).

Remark 1.1 One example where this theorem applies is when $\Omega=B(0,1)$ and $f(x, t)=$ $a(|x|) e^{p t}$ and $g(x, t)=b(|x|) h(t)$. Here $a$ and $b$ are $C^{1}$-functions with $a^{\prime}(r), b^{\prime}(r) \leq 0$ if $r \in[1-\varepsilon, 1]$ and $h: \mathbb{R} \rightarrow \mathbb{R}^{+}$is any continuous function.

In order to obtain a priori $i$ bounds for the solutions of system (1.1) we have to assume further conditions regarding the growth at infinity of the nonlinearities $f$ and $g$. For that matter we introduce the following conditions:

$\left(H_{4}\right) f(x, t) \leq c e^{t^{\alpha}}$, for some constants $c>0$ and $\alpha>0$,

$\left(H_{4}^{\prime}\right) g(x, t) \leq c e^{t^{\beta}}$, for some constants $c>0$ and $\beta>0$.

For the first result on a priori estimates of solutions of system (1.1), we consider nonlinearities satisfying $\left(H_{4}\right)$ and $\left(H_{4}^{\prime}\right)$, with

$$
\alpha+\beta<2 \text {. }
$$


Remark 1.2 Conditions $\left(H_{4}\right),\left(H_{4}^{\prime}\right)$ and $\left(H_{5}\right)$ will be satisfied if $f(x, t) \leq c e^{p t^{\alpha^{\prime}}}$ and $g(x, t) \leq c e^{q t^{\beta^{\prime}}}$, for any constants $p, q>0$, where $c>0$ and $\alpha^{\prime}, \beta^{\prime}>0$ with $\alpha^{\prime}+\beta^{\prime}<2$.

Theorem 1.3 Assume $\left(H_{4}\right),\left(H_{4}^{\prime}\right)$ and $\left(H_{5}\right)$. Then there exists a constant $C>0$ such that

$$
\|u\|_{L^{\infty}} \leq C \text { and }\|v\|_{L^{\infty}} \leq C
$$

for all eventual solutions $(u, v)$ of system (1.1).

Note that hypothesis $\left(H_{5}\right)$ allows that one nonlinearity has a growth faster than the pure exponential, provided the other nonlinearity "compensates" with a suitable growth lower than the pure exponential. The proof of this theorem is quite direct, using a Hölder type inequality in a suitable Orlicz space setting.

The next theorems concern a limiting case of Theorem 1.3, namely the case when both nonlinearities have at most exponential growth: $f(x, t), g(x, t) \leq c_{1} e^{t}$; that is, we have $\alpha=\beta=1$.

Remark 1.3 The limiting cases $\alpha \neq \beta, \alpha+\beta=2$, remain open.

Our method of proving the two results below requires that one of the nonlinearities should have a precise exponential growth. Namely, one of the next two assumptions should hold:

$\left(H_{6}\right) f(x, t) \geq c e^{t}$, for some constant $c>0$,

$\left(H_{6}^{\prime}\right) g(x, t) \geq c e^{t}$, for some constant $c>0$.

Theorem 1.4 (A priori bounds - convex domain) Assume $\left(H_{4}\right)$ and $\left(H_{4}^{\prime}\right)$ with $\alpha=$ $\beta=1$, and either $\left(H_{6}\right)$ or $\left(H_{6}^{\prime}\right)$. Assume furthermore that $\Omega$ is convex and that $\left(\mathrm{H}_{2}\right)$ holds. Then there exists a constant $\mathrm{C}>0$ such that

$$
\|u\|_{L^{\infty}} \leq C \text { and }\|v\|_{L^{\infty}} \leq C
$$

for all eventual solutions $(u, v)$ of system (1.1).

Theorem 1.5 (A priori bounds - general domain) Assume $\left(H_{4}\right)$ and $\left(H_{4}^{\prime}\right)$ with $\alpha=$ $\beta=1$, and either $\left(H_{6}\right)$ or $\left(H_{6}^{\prime}\right)$. Furthermore, assume $\left(H_{3}\right)$. Then there exists a constant $C>0$ such that

$$
\|u\|_{L^{\infty}} \leq C \text { and }\|v\|_{L^{\infty}} \leq C
$$

for all eventual solutions $(u, v)$ of system (1.1).

Remark 1.4 If the nonlinearities satisfy both conditions $\left(H_{1}\right)$ and $\left(H_{1}^{\prime}\right)$ for some constants $p, q>0$, we make a change of the variables $z=q v$ and $w=p u$ and the new equations satisfy conditions $\left(H_{4}\right)$ and $\left(H_{4}^{\prime}\right)$ with $\alpha=\beta=1$. 
Remark 1.5 It is well known that in the use of Topological methods (Leray-Schauder degree theory) for the existence of solutions of elliptic equations or systems, the main difficulty lies in obtaining a priori bounds for solutions. This is our main concern in the present paper. With some natural assumptions on the nonlinearities $f(x, t)$ and $g(x, t)$ near $t=0$ one can prove that a certain Leray-Schauder index is not zero. The a priori bound proves that the Leray-Schauder index in a large ball is zero. Therefore, by excision, one proves that there exists a non trivial solution.

Remark 1.6 Existence of solutions for systems like (1.1) in dimension two has been studied before by variational methods under a different set of assumptions on the nonlinearities $f(x, t)$ and $g(x, t)$ (see [8], [9], [11]). Observe that here we are dealing with non autonomous problems that, as far as we know, have not been considered before.

For the proofs of Theorems 1.4 and 1.5 we adapt to the case of systems the methods introduced by Brezis-Merle [4] to treat the scalar case. This will be done in section 5 .

\section{Regularity of distribution solutions}

Next, for easy reference, we state a result due to Brezis-Merle [4] which will be used to prove Theorem 1.1 above, and also in the proofs of Theorems 1.4 and 1.5.

Proposition 2.1 (Brezis-Merle) Let $u$ be a distribution solution of the linear equation

$$
\left\{\begin{aligned}
-\Delta u & =h(x) & & \text { in } \Omega \\
u & =0 & & \text { on } \partial \Omega,
\end{aligned}\right.
$$

where $\Omega$ is a bounded domain in $\mathbb{R}^{2}$, and $h \in L^{1}(\Omega)$. Then

i) for every $\delta \in(0,4 \pi)$, we have

$$
\int_{\Omega} \exp \left[\frac{(4 \pi-\delta)|u(x)|}{\|h\|_{L^{1}}}\right] d x \leq \frac{4 \pi^{2}}{\delta}(\operatorname{diam} \Omega)^{2} .
$$

ii) for every $k>0, e^{k u} \in L^{1}(\Omega)$.

Proof of Theorem 1.1. Let $(u, v)$ be a given solution of (1.1). Let us assume condition $\left(H_{1}^{\prime}\right)$. Since $-\Delta v=f(x, u)$ in $\Omega, v=0$ on $\partial \Omega$ and $f(x, u)$ belongs to $L^{1}(\Omega)$, it follows from Proposition 2.1 that

$$
\int_{\Omega} e^{k v} d x<\infty, \forall k>0
$$

Next, using the other equation in (1.1), namely $-\Delta u=g(x, v)$ in $\Omega, u=0$ on $\partial \Omega$, together with the assumption $\left(H_{1}^{\prime}\right)$, we conclude that $u \in W^{2, p}(\Omega)$ for every $p>1$. Therefore, $u \in L^{\infty}(\Omega)$. Finally, coming back to the equation $-\Delta v=f(x, u)$, we conclude that also $v \in L^{\infty}(\Omega)$. Using similar arguments we come to the same conclusions, if $\left(H_{1}\right)$ is assumed instead of $\left(H_{1}^{\prime}\right)$.

Remark 2.1 As a consequence of Theorem 1.1 and standard regularity results for elliptic equations we have that solutions of (1.1) in the distribution sense are, in fact, classical solutions. 


\section{Proof of Theorem 1.2 (uniform estimates)}

Let $\varphi_{1}$ be an eigenfunction associated to the first eigenvalue $\lambda_{1}$ of $\left(-\Delta, H_{0}^{1}(\Omega)\right)$, which is chosen in such a way that $\varphi_{1}>0$ and $\int_{\Omega} \varphi_{1}^{2}=1$.

Lemma 3.1 For each $(u, v)$ solution of system (1.1) we have

$$
\int_{\Omega} g(x, v) \varphi_{1} d x \leq C, \int_{\Omega} f(x, u) \varphi_{1} d x \leq C,
$$

where the constant $C$ depends only on $f, g$ and $\Omega$.

Proof. From our basic assumptions (i) and (ii) it follows that, given $\varepsilon>0$, there is a constant $c>0$ such that

$$
f(x, t) \geq\left(a_{1}-\varepsilon\right) t-c \text { and } g(x, t) \geq\left(a_{2}-\varepsilon\right) t-c .
$$

Next, multiplying the equations in (1.1) by $\varphi_{1}$, integrating by parts and using (3.2), we obtain

$$
\begin{aligned}
& \int_{\Omega} g(x, v) \varphi_{1} d x=\lambda_{1} \int_{\Omega} u \varphi_{1} d x \geq\left(a_{1}-\varepsilon\right) \int_{\Omega} v \varphi_{1} d x-c_{1} \\
& \int_{\Omega} f(x, u) \varphi_{1} d x=\lambda_{1} \int_{\Omega} v \varphi_{1} d x \geq\left(a_{2}-\varepsilon\right) \int_{\Omega} u \varphi_{1} d x-c_{1} .
\end{aligned}
$$

Thus

$$
\lambda_{1} \int_{\Omega} u \varphi_{1} d x \geq \frac{\left(a_{1}-\varepsilon\right)\left(a_{2}-\varepsilon\right)}{\lambda_{1}} \int_{\Omega} u \varphi_{1} d x-c_{1}
$$

which implies

$$
\int_{\Omega} u \varphi_{1} d x \leq C
$$

and therefore,

$$
\int_{\Omega} g(x, v) \varphi_{1} d x \leq C .
$$

The other inequality in (3.1) is obtained in a similar way.

Lemma 3.2 Assume condition $\left(H_{2}\right)$ and $\Omega$ convex. Then there exist $r, \delta>0$ such that

$$
\nabla u(x) \cdot \theta \leq 0 \text { and } \nabla v(x) \cdot \theta \leq 0 \text { for all } x \in \Omega_{r},|\theta-\nu(x)|<\delta,
$$

for each $(u, v)$ solution of (1.1), where $\Omega_{r}$ is defined in (1.2); $\theta$ and $\nu$ are as in $\left(H_{2}\right)$.

Proof. We can assume, without loss of generality, that $\Omega \subset \mathbb{R}_{+}^{2}:=\left\{(x, y) \in \mathbb{R}^{2}: x>0\right\}$ and $(0,0) \in \partial \Omega$. Now, we consider $T_{\lambda}:=\{(x, y): x=\lambda\}$, the cap $\Sigma_{\lambda}:=\{(x, y) \in \Omega$ : $x<\lambda\}$ and reflected cap $\Sigma_{\lambda}^{\prime}:=\left\{(2 \lambda-x, y):(x, y) \in \Sigma_{\lambda}\right\}$. It follows that there exists $\bar{\lambda}$ such that $\Sigma_{\lambda} \cup \Sigma_{\lambda}^{\prime} \subset \Omega_{r}$ for each $0<\lambda<\bar{\lambda}$. In fact this $\bar{\lambda}$ depends only on $r$ and not on the particular point on the boundary. 
For $0<\lambda<\bar{\lambda}$, define in $\Sigma_{\lambda}$ the auxiliary functions

$$
\begin{aligned}
w_{\lambda}(x, y) & =u(2 \lambda-x, y)-u(x, y), \\
z_{\lambda}(x, y) & =v(2 \lambda-x, y)-v(x, y) .
\end{aligned}
$$

Using condition $\left(H_{3}\right)$ we have

$$
\begin{aligned}
\Delta w_{\lambda} & =-g((2 \lambda-x, y), v(2 \lambda-x, y))+g((x, y), v(x, y)) \\
& \leq-g((x, y), v(2 \lambda-x, y))+g((x, y), v(x, y))
\end{aligned}
$$

Now, using the Mean Value Theorem wee see that

$$
\Delta w_{\lambda} \leq c(x, y)(v(x, y)-v(2 \lambda-x, y)),
$$

where

$$
c(x, y)=\frac{\partial g}{\partial t}((x, y), \eta(x, y)) \geq 0
$$

and $\eta(x, y)$ is a real number between $v(x, y)$ and $v(2 \lambda-x, y)$. Thus

$$
\Delta w_{\lambda}+c(x, y) z_{\lambda} \leq 0
$$

Similarly we can prove that

$$
\Delta z_{\lambda}+\widetilde{c}(x, y) w_{\lambda} \leq 0
$$

where

$$
\widetilde{c}(x, y)=\frac{\partial f}{\partial t}((x, y), \zeta(x, y)) \geq 0
$$

and $\zeta(x, y)$ is a real number between $u(x, y)$ and $u(2 \lambda-x, y)$.

For $\lambda$ sufficiently small and positive we have that $\Sigma_{\lambda}$ has small measure, and so we can use the Maximum Principle for cooperative elliptic systems in small domains (see [6] and [2]) to conclude that

$$
w_{\lambda} \geq 0 \text { and } z_{\lambda} \geq 0 \text { in } \Sigma_{\lambda} .
$$

Using similar arguments as in [6] we can also prove that

$$
w_{\lambda} \geq 0 \text { and } z_{\lambda} \geq 0 \text { in } \Sigma_{\bar{\lambda}} .
$$

Therefore, there exists $\epsilon>0$ such that $u$ and $v$ are increasing in $\Omega_{\epsilon}$. Finally, the conclusion follows in a standard way as in [7].

The next two lemmas are straightforward adaptations to the case of systems of well known results in the scalar case.

Lemma 3.3 Assume condition $\left(H_{3}\right)$. Then the same conclusion of Lemma 3.2 holds.

Proof. The use of Moving Planes as in the previous proof is possible after using a Kelvin transform about the points on the boundary where the domain is not convex, see [7] and [5]. Condition $\left(\mathrm{H}_{3}\right)$ implies that the transformed equations have nonlinearities with right monotonicity near the boundary, see details in [5]. 
Lemma 3.4 Assume the hypotheses of either Lemma 3.2 or Lemma 3.3. Then there exist $\varepsilon>0$ and $C>0$ which depend only on $f, g$ and $\Omega$ such that $\|u\|_{L^{\infty}\left(\Omega_{\varepsilon}\right)},\|v\|_{L^{\infty}\left(\Omega_{\varepsilon}\right)} \leq C$, for each $(u, v)$ solution of (1.1).

Proof. The proof follows by the same arguments as in [7] (see Step 2 in the proof of Theorem 1.1, page 45 of [7]), using Lemmas 3.2 and 3.3 above.

Now, we complete the proof of Theorem 1.2. Let $a:=\inf \left\{\varphi_{1}(x): x \in \bar{\Omega} \backslash \Omega_{\varepsilon}\right\}$. Using Lemma 3.4 we obtain that $f(x, v)$ is bounded in $\Omega_{\varepsilon}$. Thus

$$
\begin{aligned}
\int_{\Omega} f(x, v) d x & =\int_{\Omega_{\varepsilon}} f(x, v) d x+\int_{\Omega \backslash \Omega_{\varepsilon}} f(x, v) d x \\
& \leq C+\frac{1}{a} \int_{\Omega \backslash \Omega_{\varepsilon}} \varphi_{1} f(x, v) d x \\
& =C+\frac{1}{a} \int_{\Omega} \varphi_{1} f(x, v) d x \leq C,
\end{aligned}
$$

where we have used Lemma 3.1 to estimate the last integral. Using a similar argument we can prove the result for $g(x, u)$.

\section{Proof of Theorem 1.3}

In this section we rely on an inequality which was introduced in [8] to treat elliptic systems in dimension two; it is a sort of Young's inequality.

\section{Proposition 4.1}

$$
s t \leq \begin{cases}e^{t^{2}}-1+s\left(\log ^{+} s\right)^{1 / 2}, & t \geq 0, s \geq e^{1 / 4} \\ e^{t^{2}}-1+\frac{1}{2} s^{2}, & t \geq 0,0 \leq s \leq e^{1 / 4}\end{cases}
$$

First we recall that Theorem 1.1 gives that each $(u, v)$ solution of (1.1) belongs to $\left(L^{\infty}(\bar{\Omega})\right)^{2}$, and then it follows that it belongs to $\left(W^{1,2}(\Omega)\right)^{2}$. Using the first equation of system (1.1) we obtain

$$
\int_{\Omega}|\nabla u|^{2} d x=\int_{\Omega} u g(x, v) d x .
$$

Now, by Proposition 4.1, with $t=\frac{u}{\|u\|} \sqrt{4 \pi}$ and $s=g(x, v)$, we get

$$
\begin{aligned}
\int_{\Omega} u g(x, v) d x= & \frac{\|u\|}{\sqrt{4 \pi}} \int_{\Omega} \frac{u}{\|u\|} \sqrt{4 \pi} g(x, v) d x \\
\leq & \frac{\|u\|}{\sqrt{4 \pi}}\left\{\int_{\Omega} e^{4 \pi\left(\frac{u}{\|u\|}\right)^{2}} d x-|\Omega|+\int_{\Omega} g(x, v)\left(\log ^{+} g(x, v)\right)^{1 / 2} d x+\right. \\
& \left.+\frac{1}{2} \int_{\left\{g(x, v(x)) \leq e^{1 / 4}\right\}} g(x, v)^{2} d x\right\} \\
\leq & \frac{\|u\|}{\sqrt{4 \pi}}\left\{C+\int_{\Omega} g(x, v)\left(\log ^{+} g(x, v)\right)^{1 / 2} d x\right\}
\end{aligned}
$$


where in the last estimate we have used the Trudinger-Moser inequality (see [1] and [10]).

So we have proved that

$$
\|u\| \leq \frac{1}{\sqrt{4 \pi}}\left\{C+\int_{\Omega} g(x, v)\left(\log ^{+} g(x, v)\right)^{1 / 2} d x\right\} .
$$

Since $g(x, t) \leq c e^{d v^{\beta}}$, we have $\log ^{+} g(x, v) \leq \log c+d v^{\beta}$ and $\left(\log ^{+} g(x, v)\right)^{1 / 2} \leq$ $c+d^{1 / 2} v^{\beta / 2}$. So,

$$
\begin{aligned}
\int_{\Omega} g(x, v)\left(\log ^{+} g(x, v)\right)^{1 / 2} d x & \leq \int_{\Omega} c g(x, v) d x+d^{1 / 2} \int_{\Omega} g(x, v) v^{\beta / 2} d x \\
& \leq C\left(1+\int_{\Omega} \chi_{[v \geq 1]} g(x, v) v^{\beta / 2} d x\right),
\end{aligned}
$$

where we have used Theorem 1.2. It remains to estimate $\int_{\Omega} \chi_{[v \geq 1]} g(x, v) v^{\beta / 2} d x$. For that matter we use Hölder's inequality in Orlicz spaces (see [1]) for the Young pair:

$$
\varphi(t):=e^{t^{\gamma}} \text { and } \psi(s):=s\left((\log s)^{1 / \gamma}-1\right),
$$

where $\gamma>0$ will be chosen later. So we can proceed as follows:

$$
\begin{aligned}
\int_{\Omega} \chi_{[v \geq 1]} g(x, v) v^{\beta / 2} d x & =\int_{\Omega} \chi_{[v \geq 1]} \frac{g(x, v)}{(v+1)^{\eta}} v^{\beta / 2}(v+1)^{\eta} d x \\
& \leq\left\|\chi_{[v \geq 1]} v^{\beta / 2}(v+1)^{\eta}\right\|_{L^{\varphi}}\left\|\frac{g(x, v)}{(v+1)^{\eta}}\right\|_{L^{\psi}},
\end{aligned}
$$

where $\eta>0$ will also be chosen later. Recall that $\|\cdot\|_{L^{\varphi}}$ stands for the gauge norm in the Orlicz space $L^{\varphi}$, which is defined as follows

$$
\|u\|_{L^{\varphi}}=\inf \left\{k>0: \int_{\Omega} \varphi\left(\frac{u}{k}\right) \leq 1\right\} .
$$

Next we estimate the two gauge norms:

$$
\left\|\chi_{[v \geq 1]} v^{\beta / 2}(v+1)^{\eta}\right\|_{L^{\varphi}} \leq c\left\|v^{\beta / 2+\eta}\right\|_{L^{\varphi}}=\inf \left\{k>0: \int_{\Omega} e^{\left(\frac{v^{\eta+\beta / 2}}{k}\right)^{\gamma}} \leq 1\right\} .
$$

Now, viewing to use Trudinger-Moser estimate, $\gamma$ and $\eta$ should satisfy $\gamma(\eta+\beta / 2)=2$. Then, if we take $k=\|v\|^{2 / \gamma}$, we have $\int_{\Omega} e^{\frac{v^{2}}{k \gamma}} \leq$ const, and this implies

$$
\left\|\chi_{[v \geq 1]} v^{\beta / 2}(v+1)^{\eta}\right\|_{L^{\varphi}} \leq c\|v\|^{2 / \gamma} .
$$

Now we estimate the other gauge norm:

$$
\left\|\frac{g(x, v)}{(v+1)^{\eta}}\right\|_{L^{\psi}}=\inf \left\{k: \int_{\Omega} \frac{g(x, v)}{k(v+1)^{\eta}}\left[\left(\log ^{+} \frac{g(x, v)}{k(v+1)^{\eta}}\right)^{1 / \gamma}-1\right] d x \leq 1\right\}
$$

Notice that

$$
\frac{g(x, v)}{(v+1)^{\eta}} \leq C e^{d v^{\beta}}
$$


which implies that

$$
\left(\log ^{+} \frac{g(x, v)}{k(v+1)^{\eta}}\right)^{1 / \gamma} \leq C+d^{1 / \gamma} v^{\beta / \gamma} .
$$

Continuing the estimate of the integral in (4.5) we obtain

$$
\frac{g(x, v)}{k(v+1)^{\eta}}\left[\left(\log ^{+} \frac{g(x, v)}{k(v+1)^{\eta}}\right)^{1 / \gamma}\right] \leq \frac{C g(x, v)}{k(v+1)^{\eta}}+\frac{g(x, v)}{k(v+1)^{\eta}} d^{1 / \gamma} v^{\beta / \gamma} \leq \frac{C g(x, v)}{k},
$$

if we choose $\eta=\beta / \gamma$. Thus,

$$
\left\|\frac{g(x, v)}{(v+1)^{\eta}}\right\|_{L^{\psi}} \leq C \int_{\Omega} g(x, v) d x \leq C .
$$

Finally, using (4.1), (4.4) and (4.6) we have

$$
\|u\| \leq C \frac{1}{\sqrt{4 \pi}}\left\{C+\|v\|^{2 / \gamma}\right\}
$$

where $\gamma$ and $\eta$ have to be chosen in order to satisfy the two conditions above, namely $\gamma(\eta+\beta / 2)=2$ and $\eta=\beta / \gamma$. So $\gamma=2(2-\beta) / \beta$.

Using an argument similar to the one we have just completed, we can prove

$$
\|v\| \leq C \frac{1}{\sqrt{4 \pi}}\left\{C+\|u\|^{2 / \gamma^{\prime}}\right\}
$$

where $\gamma^{\prime}=2(2-\alpha) / \alpha$.

It follows then from (4.7) and (4.8) that

$$
\|u\| \leq C\left(C+\|u\|^{\frac{4}{\gamma \gamma^{\prime}}}\right) .
$$

So, in order to conclude the proof of Theorem 1.3, we observe that the condition $\alpha+\beta<2$ implies that $4 /\left(\gamma \gamma^{\prime}\right)<1$.

\section{Proofs of Theorems 1.4 and 1.5}

Once the uniform estimates in Theorem 1.2 are established, the proofs of Theorems 1.4 and 1.5 are the same. So from now on we assume conditions $\left(H_{4}\right)$ and $\left(H_{4}^{\prime}\right)$ with $\alpha=\beta=1$.

Assume that $\left(\left(u_{n}, v_{n}\right)\right)$ is a sequence of solutions of (1.1). Theorem 1.2 says that the sequences $\left(f\left(x, v_{n}\right)\right)$ and $\left(g\left(x, u_{n}\right)\right)$ are bounded in $L^{1}(\Omega)$. So it follows, passing to subsequences if necessary, (see [3]) that there are measures $\mu$ and $\nu$ such that

$$
f\left(x, u_{n}\right) \rightarrow \mu \text { and } g\left(x, v_{n}\right) \rightarrow \nu .
$$

Since $f$ and $g$ are positive functions it follows that $\mu$ and $\nu$ are nonnegative measures. We also observe that, as a consequence of Theorem 1.2, the solutions $\left(\left(u_{n}, v_{n}\right)\right)$ of $(1.1)$ are bounded in $L^{1}(\Omega)$ :

$$
\left\|u_{n}\right\|_{L^{1}},\left\|v_{n}\right\|_{L^{1}} \leq C, \forall n
$$


Definition 5.1 We say that $x_{0} \in \Omega$ is a regular point of the measure $\mu$ if there is a function $\psi \in C_{c}(\Omega), 0 \leq \psi \leq 1$, with $\psi \equiv 1$ in some neighborhood of $x_{0}$ and such that

$$
\int \psi d \mu<4 \pi
$$

We denote by $\Sigma_{\mu}$ the set of non-regular points in $\Omega$ for the measure $\mu$.

Remark 5.1 For a bounded non-negative measure $\mu, \Sigma_{\mu}$ is a finite set. Indeed, if $x_{0} \in \Sigma_{\mu}$, we have that

$$
\int \psi d \mu \geq 4 \pi
$$

for each function $\psi \in C_{c}(\Omega), 0 \leq \psi \leq 1$, with $\psi \equiv 1$ in some neighborhood of $x_{0}$. Thus, $\mu\left(\left\{x_{0}\right\}\right) \geq 4 \pi$. Finally, since $\int d \mu \leq C$, it follows that $\Sigma_{\mu}$ is a finite set.

Let $S_{u}$ be the blow-up set for the sequence $\left(u_{n}\right)$, that is

$$
S_{u}:=\left\{x \in \Omega: \exists\left(x_{n}\right) \subset \Omega \text { such that } x_{n} \rightarrow x \text { and } u_{n}\left(x_{n}\right) \rightarrow+\infty\right\} .
$$

The assertions of Theorems 1.4 and 1.5 will be proved if we show that $S_{u}=S_{v}=\emptyset$. This will be achieved in the next lemmas.

Lemma 5.1 Assume that $x_{0}$ is a regular point for the measure $\mu$ (or for the measure $\nu)$. Then there exist constants $\rho>0$ and $C$, independent of $n$, such that

$$
\left\|u_{n}\right\|_{L^{\infty}\left(B_{\rho}\left(x_{0}\right)\right)} \leq C,\left\|v_{n}\right\|_{L^{\infty}\left(B_{\rho}\left(x_{0}\right)\right)} \leq C .
$$

Proof. Using the fact that $x_{0}$ is a regular point of the measure $\mu$ we have a function $\psi \in C_{c}(\Omega), 0 \leq \psi \leq 1$, with $\psi \equiv 1$ in some neighborhood $V_{x_{0}}$ of $x_{0}$, such that $\int \psi d \mu<4 \pi$. Thus, $\int_{V_{x_{0}}} d \mu<4 \pi$, which implies that there exist $R>0, \delta>0$ and $n_{0}$ such that for all $n \geq n_{0}$

$$
\int_{B_{R}\left(x_{0}\right)} f\left(x, u_{n}\right) d x \leq 4 \pi-\delta
$$

Using this estimate, we first work with the second equation in (1.1). Let us write $v_{n}:=v_{1, n}+v_{2, n}$, where

$$
-\Delta v_{1, n}=f\left(x, u_{n}\right), \text { in } B_{R}\left(x_{0}\right) \text { and } v_{1, n}=0 \text { for }\left|x-x_{0}\right|=R .
$$

Notice that $-\Delta v_{2, n}=0$ in $B_{R}\left(x_{0}\right)$.

Using Proposition 2.1 and (5.3), we obtain

$$
c \geq \int_{B_{R}} e^{\left(4 \pi-\frac{\delta}{2}\right) \frac{v_{1, n}}{\int|f|}} \geq \int_{B_{R}} e^{\left(4 \pi-\frac{\delta}{2}\right) \frac{v_{1, n}}{4 \pi-\delta}}=\int_{B_{R}} e^{p v_{1, n}},
$$

where $p>1$ is a constant depending only on $\delta$. Using the fact that $t \leq e^{t}$ we get

$$
\left\|v_{1, n}\right\|_{L^{p}\left(B_{R}\left(x_{0}\right)\right)} \leq C
$$


Since the function $v_{2, n}$ is harmonic in $B_{R}\left(x_{0}\right)$, it follows from the Mean Value Theorem for harmonic functions that

$$
\left\|v_{2, n}\right\|_{L^{\infty}\left(B_{R / 2}\right)} \leq C\left\|v_{2, n}\right\|_{L^{1}\left(B_{R}\right)} .
$$

On the other hand, using (5.2) and (5.5) we obtain

$$
\left\|v_{2, n}\right\|_{L^{1}\left(B_{R}\right)} \leq\left\|v_{n}\right\|_{L^{1}\left(B_{R}\right)}+\left\|v_{1, n}\right\|_{L^{1}\left(B_{R}\right)} \leq C
$$

and so

$$
\left\|v_{2, n}\right\|_{L^{\infty}\left(B_{R / 2}\right)} \leq C
$$

Let us now use assumption $\left(H_{4}^{\prime}\right)$ with $\beta=1$. Then $g\left(x, v_{n}\right) \leq c e^{v_{1, n}+v_{2, n}}=$ $c e^{v_{1, n}} e^{v_{2, n}}$. So it follows from (5.4) and (5.6) that

$$
\left\|g\left(x, v_{n}\right)\right\|_{L^{p}\left(B_{R / 2}\left(x_{0}\right)\right)} \leq c\left\|e^{v_{n}}\right\|_{L^{p}\left(B_{R / 2}\left(x_{0}\right)\right)} \leq C, \text { for some } p>1 .
$$

In order to prove that $\left\|v_{n}\right\|_{L^{\infty}\left(B_{\rho}\left(x_{0}\right)\right)} \leq C$, for some $\rho<R / 2$, it is now enough to prove a similar bound as (5.6) for $v_{1, n}$, namely

$$
\left\|v_{1, n}\right\|_{L^{\infty}\left(B_{\rho}\left(x_{0}\right)\right)} \leq C .
$$

For that matter, we use the first equation in (1.1). Let us write $u_{n}=u_{1, n}+u_{2, n}$ where

$$
-\Delta u_{1, n}=g\left(x, v_{n}\right) \text {, in } B_{R / 2}\left(x_{0}\right) \text { and } u_{1, n}=0 \text { for }\left|x-x_{0}\right|=R / 2 .
$$

Observe that in view of (5.7), the assumption on $g$, and by elliptic regularity we have

$$
\left\|u_{1, n}\right\|_{L^{\infty}\left(B_{R / 2}\left(x_{0}\right)\right)} \leq C .
$$

Notice that $-\Delta u_{2, n}=0$ in $B_{R / 2}\left(x_{0}\right)$. Thus $u_{2, n}$ is harmonic in $B_{R / 2}\left(x_{0}\right)$, and it follows from the Mean Value Theorem for harmonic functions that

$$
\left\|u_{2, n}\right\|_{L^{\infty}\left(B_{R / 4}\right)} \leq\left\|u_{2, n}\right\|_{L^{1}\left(B_{R / 2}\right)} \leq\left\|u_{n}\right\|_{L^{1}\left(B_{R / 2}\right)}+\left\|u_{1, n}\right\|_{L^{1}\left(B_{R / 2}\right)} \leq C .
$$

From (5.9) and (5.10) we have

$$
\left\|u_{n}\right\|_{L^{\infty}\left(B_{R / 4}\right)} \leq C .
$$

Now we go back to the second equation in (1.1). We write $v_{n}:=\widetilde{v}_{1, n}+\widetilde{v}_{2, n}$, where

$$
-\Delta \widetilde{v}_{1, n}=f\left(x, u_{n}\right) \text {, in } B_{R / 4}\left(x_{0}\right) \text { and } \widetilde{v}_{1, n}=0 \text { for }\left|x-x_{0}\right|=R / 4 .
$$

Using (5.11) and elliptic regularity we have

$$
\left\|\widetilde{v}_{1, n}\right\|_{L^{\infty}\left(B_{R / 4}\right)} \leq C .
$$

Notice that $-\Delta \widetilde{v}_{2, n}=0$ in $B_{R / 4}\left(x_{0}\right)$. As before, from the Mean Value Theorem for harmonic functions we have

$$
\left\|\widetilde{v}_{2, n}\right\|_{L^{\infty}\left(B_{R / 8}\right)} \leq C
$$

From (5.12) and (5.13) we have

$$
\left\|v_{n}\right\|_{L^{\infty}\left(B_{R / 8}\right)} \leq C,
$$

which together with (5.11) proves Lemma 5.1, taking $\rho=R / 8$. 
Lemma 5.2 $S_{u} \subset \Sigma_{\mu}$ and $S_{v} \subset \Sigma_{\nu}$.

Proof. This follows directly from Lemma 5.1 and the definition of the sets $\Sigma_{\mu}, S_{u}, S_{v}$ and $\Sigma_{\nu}$

Lemma 5.3 $\Sigma_{\mu} \subset S_{v}$ and $\Sigma_{\nu} \subset S_{u}$.

Proof. Let $x_{0} \in \Sigma_{\mu}$. We claim that for each $R>0$ we have

$$
\lim _{n \rightarrow+\infty}\left\|u_{n}\right\|_{L^{\infty}\left(B_{R}\left(x_{0}\right)\right)}=+\infty .
$$

Suppose by contradiction that there exists $R_{0}>0$ and a subsequence, which we denote also by $\left(u_{n}\right)$, such that

$$
\left\|u_{n}\right\|_{L^{\infty}\left(B_{R_{0}}\left(x_{0}\right)\right)} \leq C .
$$

So,

$$
\left\|e^{u_{n}}\right\|_{L^{\infty}\left(B_{R_{0}}\left(x_{0}\right)\right)} \leq C .
$$

Now using the hypothesis $f(x, u) \leq c e^{u}$ it follows that

$$
\left\|f\left(x, u_{n}\right)\right\|_{L^{\infty}\left(B_{R_{0}}\left(x_{0}\right)\right)} \leq C,
$$

which implies that for $R<R_{0}$ we have

$$
\int_{B_{R}\left(x_{0}\right)} f\left(x, u_{n}\right) \leq C R^{2} .
$$

Thus, there exists $R_{1}>0$, such that

$$
\int_{B_{R_{1}}\left(x_{0}\right)} f\left(x, u_{n}\right)<4 \pi .
$$

This implies that $x_{0}$ is a regular point of $\mu$, which is a contradiction.

Now we observe that, using Remark 5.1, there exists $R>0$ such that $x_{0}$ is the only non-regular point in $B_{R}\left(x_{0}\right)$.

Next, we use (5.14) to prove that $x_{0} \in S_{v}$. Indeed, from (5.14) there exists $\left(x_{n}\right) \subset$ $B_{R}\left(x_{0}\right)$ such that $x_{n} \rightarrow \tilde{x}$ and $v\left(x_{n}\right) \rightarrow+\infty$. So, one needs to prove $\tilde{x}=x_{0}$. Indeed if this were not the case, then $\tilde{x}$ would be a regular point, which is not possible, since $u_{n}$ is bounded in a neighborhood of a regular point.

With similar arguments as in the proof we just completed, we can prove that $\Sigma_{\nu} \subset S_{u}$.

As a consequence of Lemmas 5.2 and 5.3 we conclude that those four sets coincide:

$$
S_{u}=\Sigma_{\mu}=S_{v}=\Sigma_{\nu} .
$$

Finally, we prove that this set is indeed empty, and this completes the proofs of the Theorems 1.4 and 1.5.

Lemma 5.4 $S_{u}=\emptyset$. 
Proof. Suppose, by contradiction, that there exists $x_{0} \in S_{u}$. Since $x_{0}$ is isolated, we can take $R>0$ such that $\overline{B_{R}\left(x_{0}\right)} \cap\left(S_{u} \backslash\left\{x_{0}\right\}\right)=\emptyset$.

Next, we consider the Dirichlet problem in $B_{R}\left(x_{0}\right)$,

$$
-\Delta z_{n}=f\left(x, u_{n}\right) \text {, in } B_{R}\left(x_{0}\right) \text { and } z_{n}=0 \text { when }\left|x-x_{0}\right|=R \text {. }
$$

We know that the function $u_{n}$ satisfies

$$
-\Delta v_{n}=f\left(x, u_{n}\right), \text { in } B_{R}\left(x_{0}\right) \text { and } u_{n} \geq 0 \text { when }\left|x-x_{0}\right|=R .
$$

Thus, by the Maximum Principle we have

$$
0 \leq z_{n} \leq v_{n} \text { in } \overline{B_{R}\left(x_{0}\right)}
$$

Taking the limit we have that $z_{n} \rightarrow z$, where $z$ is a solution of the problem

$$
-\Delta z=\mu \text {, in } B_{R}\left(x_{0}\right) \text { and } z=0 \text { when }\left|x-x_{0}\right|=R .
$$

On the other hand the problem

$$
-\Delta w=4 \pi \delta_{0} \text { in } B_{R}\left(x_{0}\right) \text { and } w=0 \text { when }\left|x-x_{0}\right|=R .
$$

has the solution

$$
w(x)=2 \log \frac{R}{\left|x-x_{0}\right|} .
$$

Since $x_{0}$ is not a regular point it follows that $\mu \geq 4 \pi \delta_{0}$. So

$$
z(x) \geq 2 \log \left|x-x_{0}\right|^{-1}+o(1), x \rightarrow x_{0}
$$

Now with the hypothesis $g(x, t) \geq C e^{t}$, we have

$$
\begin{aligned}
\lim _{n \rightarrow \infty} \int_{B_{R}\left(x_{0}\right)} g\left(x, v_{n}\right) & \geq \lim _{n \rightarrow \infty} \int_{B_{R}\left(x_{0}\right)} g\left(x, z_{n}\right) \\
& \geq \int_{B_{R}\left(x_{0}\right)} g(x, z) \\
& \geq c \int_{B_{R}\left(x_{0}\right)} e^{z} \\
& \geq c \int_{B_{R}\left(x_{0}\right)} e^{w}=\infty
\end{aligned}
$$

which is impossible.

\section{References}

[1] R.A. Adams, J.F. Fournier, Sobolev spaces, second ed., Academic Press, (2003). 
[2] H. Berestycki, L. Nirenberg, On the method of moving planes and the sliding method, Bol. Soc. Brasil. Mat. (N.S.) 22 (1991), 1-37.

[3] H. Brezis, Analyse fonctionnelle, Théorie et applications, Masson, Paris, 1983.

[4] H. Brezis and F. Merle, Uniform estimates and blow-up behavior for solutions of $-\Delta u=V(x) e^{u}$ in two dimensions, Comm. Partial Differential Equations 16 (1991), 12231253.

[5] Wen Xiong Chen and Congming Li, A priori estimates for solutions to nonlinear elliptic equations, Arch. Rational Mech. Anal. 122 (1993), 145-157.

[6] D. G. de Figueiredo, Monotonicity and symmetry of solutions of elliptic systems in general domains, NoDEA Nonlinear Differential Equations Appl. 1 (1994), 119-123.

[7] D. G. de Figueiredo, P.-L Lions, R.D. Nussbaum, A priori estimates and existence of positive solutions of semilinear elliptic equations, J. Math. Pures Appl. 61 (1982), 41-63.

[8] D.G. de Figueiredo, João Marcos do Ó and Bernhard Ruf, Critical and subcritical elliptic systems in dimension two, Indiana Univ. Math. J. 53 (2004), 1037-1054.

[9] D. G. de Figueiredo, João Marcos do Ó and Bernhard Ruf, An Orlicz-space approach to superlinear elliptic systems, J. Funct. Anal. 224 (2005), no. 2, 471-496.

[10] J. Moser, A sharp form of an inequality by N. Trudinger, Ind. Univ. J. 20 (1971), 10771092.

[11] B. Ruf, Lorentz spaces and nonlinear elliptic systems. Contributions to Nonlinear Analysis, 471-489, Progr. Nonlinear Differential Equations Appl., 66, Birkhäuser, Basel, 2006.

[12] W. Troy, Symmetry properties in systems of semilinear elliptic equations, J. Differential Equations 42 (1981), 400-413. 